\title{
RICCI CURVATURE DECAY ON OPEN MANIFOLDS
}

\author{
DAVID WRAITH
}

\begin{abstract}
The behaviour of the Ricci curvature along rays in a complete open manifold is examined.
\end{abstract}

\section{Introduction}

The object of this paper is to make an observation concerning the behaviour of the Ricci curvature on a complete open manifold.

Theorem A. Let $M^{n}$ be a complete, non-compact Riemannian manifold, and let $\gamma(t), t \geqslant 0$, be a ray in $M$. Suppose that $\operatorname{Ric}(\dot{\gamma}(t)) \geqslant 0$ for all $t$. For $\delta>0$, let

$$
I_{\delta}=\{t>0: \operatorname{Ric}(\dot{\gamma}(t)) \geqslant(n-1) \delta\} .
$$

Then $I_{\delta}$ is Lebesgue measurable, with

$$
\mu\left(I_{\delta}\right)<\frac{\pi}{2 \sqrt{\delta}} .
$$

It is easy to construct examples of manifolds (for instance, surfaces of revolution) to show both that Theorem A is sharp, and that it fails to be true if we allow any negative Ricci curvatures along $\gamma$.

It is an elementary consequence of Theorem A that on a non-compact manifold of non-negative Ricci curvature, the Ricci curvature $\operatorname{Ric}(\dot{\gamma}(t))$ along a ray $\gamma(t)$ must exhibit some form of decay. (This is of course not necessarily true if we consider directions orthogonal to $\dot{\gamma}(t)$.) The Bonnet-Myers theorem and the result of Ambrose [1] offer different perspectives on this phenomonon.

\section{Preliminary results}

Given a ray $\gamma(t)$, we study the mean curvature of the distance spheres with center $\gamma(0)$. In particular, we investigate how this quantity varies along $\gamma(t)$. Denote by $m(t)$ the mean curvature of the distance sphere of radius $t$ at the point $\gamma(t)$. The following Riccati inequality is well-known (see, for example, [2, p. 26]):

$$
m^{\prime}(t) \leqslant-\operatorname{Ric}\left(\dot{\gamma}^{\prime}(t)\right)-\frac{m^{2}(t)}{n-1} .
$$

We have equality for all $t$ in (1) if and only if we have constant Ricci curvature $(n-1) \delta$ in the direction of $\gamma$. 
Definition 1. Define the function $z(t), t \geqslant t_{0}$, by

$$
z^{\prime}(t)=-(n-1) \delta-\frac{z^{2}(t)}{n-1}, \quad z\left(t_{0}\right)=c .
$$

An elementary calculation yields the following lemma.

LEMMA 1. If $\delta>0$, then $z(t)$ is given by

$$
z(t)=(n-1) \sqrt{\delta} \tan \left\{\tan ^{-1}\left(\frac{c}{(n-1) \sqrt{\delta}}\right)+\sqrt{\delta}\left(t_{0}-t\right)\right\} .
$$

In the case $\delta=0$, the corresponding expression is

$$
z(t)=\left(c^{-1}+\frac{t-t_{0}}{n-1}\right)^{-1} .
$$

Lemma 2. Suppose that $\operatorname{Ric}(\dot{\gamma}(t)) \geqslant(n-1) \delta$ for all $t \geqslant 0$, and that the mean curvature $m(t)$ along $\gamma(t)$ satisfies $m\left(t_{0}\right)=c$ for some $t_{0} \geqslant 0$. Using this value of $c$ in the definition of $z(t)$, for all $t \geqslant t_{0}$, we have

$$
m(t) \leqslant z(t) .
$$

Proof. This follows from the Riccati inequality (1) by a standard argument.

COROLlaRY 1. If $\operatorname{Ric}(\dot{\gamma}(t)) \geqslant(n-1) \delta$ for $t \geqslant 0$, then:

if $\delta>0$, we have $m \leqslant(n-1) \sqrt{\delta} \tan (\pi / 2-t \sqrt{\delta})$;

if $\delta=0$, we have $m \leqslant(n-1) / t$.

Proof. These formulae follow easily from Lemmas 1 and 2 by setting $t_{0}=0$ and using the fact that $\lim _{t \rightarrow 0^{+}} m(t)=\infty$.

LEMmA 3. If $\operatorname{Ric}(\dot{\gamma}(t)) \geqslant 0$ for all $t \geqslant 0$, and there exists $t_{0}>0$ for which $m\left(t_{0}\right)<0$, then there exists $t_{1}$ with $t_{0} \leqslant t_{1}<\infty$ such that

$$
\lim _{t \rightarrow t_{1}^{-}} m(t)=-\infty \text {. }
$$

Proof. In the definition of $z(t)$, let the value of $c$ be $m\left(t_{0}\right)$, and let the value of $\delta$ be 0 . By Lemma 1 we have

$$
z(t)=\left(c^{-1}+\frac{t-t_{0}}{n-1}\right)^{-1} .
$$

Now $c^{-1}<0$ and $\left(t-t_{0}\right) /(n-1)$ increases linearly from 0 for $t \geqslant t_{0}$. We see that $z(t)$ is undefined when $t=t_{0}-(n-1) c^{-1}$.

Set $t_{2}=t_{0}-(n-1) z\left(t_{0}\right)^{-1}$. As $z(t)<0$ for all $t$, we must have

$$
\lim _{t \rightarrow t_{2}^{-}} z(t)=-\infty \text {. }
$$

Lemma 2 asserts that $m(t) \leqslant z(t)$ for all $t \geqslant t_{0}$ for which $m(t)$ is defined. It follows that there exists $t_{1} \in\left[t_{0}, t_{2}\right]$ with $\lim _{t \rightarrow t_{1}^{-}} m(t)=-\infty$, as required.

Consider a geodesic $\gamma(t)$. Let $\left\{e_{i}\right\}$ be any choice of orthonormal basis for the space $\dot{\gamma}(0)^{\perp}$. By parallel translation of this frame along $\gamma$ we obtain vector fields $e_{i}(t)$ 
which form an orthonormal basis for $\dot{\gamma}(t)^{\perp}$ for all $t$. Let $J_{i}(t)$ be the (unique) Jacobi field along $\gamma(t)$ satisfying

$$
J_{i}(0)=0, \quad J_{i}^{\prime}(0)=e_{i} .
$$

(See [3] for a detailed account of Jacobi fields.)

Definition 2. Let the matrix $A_{t}$ be given by

$$
A_{t}:=\left(J_{1}\left|J_{2}\right| \ldots \mid J_{n-1}\right),
$$

where we are using the frame field $\left\{e_{i}(t)\right\}$ to express the $J_{i}$ as column vectors.

Lemma 4. Consider the geodesic $\gamma(t)$, and suppose that there is no point on $\gamma$ which is conjugate to $\gamma(0)$. Let $S_{t}$ denote the shape operator at $\gamma(t)$ of the distance sphere of radius $t$ about $\gamma(0)$. If $\tau_{t}$ denotes parallel translation along $\gamma$ from $\gamma(0)$ to $\gamma(t)$, and $\alpha_{t}$ is the linear endomorphism of $T_{\gamma(0)} M$ for which the matrix with respect to the basis $\left\{e_{i}\right\}$ is $A_{t}$, then

$$
S_{t}=\tau_{t} \alpha_{t}^{\prime} \alpha_{t}^{-1} \tau_{t}^{-1} .
$$

Note. It is clear that $\operatorname{det} A_{t} \neq 0$ (in the absence of conjugate points). Hence $\alpha_{t}$ is invertible.

Proof. Consider a vector $X \in \dot{\gamma}^{\perp}\left(t_{0}\right)$ for some $t_{0}$. We show that

$$
S_{t_{0}}(X)=\tau_{t_{0}} \alpha_{t_{0}}^{\prime} \alpha_{t_{0}}^{-1} \tau_{t_{0}}^{-1}(X) .
$$

By assumption, $\gamma$ contains no point conjugate to $\gamma(0)$, so there must be a vector $Y \in T_{\gamma(0)} M$ for which the Jacobi field along $\gamma$ defined by

$$
J(0)=0, \quad J^{\prime}(0)=Y,
$$

satisfies $J\left(t_{0}\right)=X$. From the definition of the matrix $A_{t}$ we have

$$
\tau_{t} \circ \alpha_{t}(Y)=J(t)
$$

It follows that

$$
\alpha_{t}(Y)=\tau_{t}^{-1}(J(t)),
$$

and so

$$
Y=\alpha_{t}^{-1}\left(\tau_{t}^{-1}(J(t))\right)
$$

for all $t$. Therefore

$$
\begin{aligned}
\alpha_{t}^{\prime} \circ \alpha_{t}^{-1}\left(\tau_{t}^{-1}(J(t))\right) & =\alpha_{t}^{\prime}(Y) \\
& =\left[\frac{d}{d t} \alpha_{t}\right](Y) \\
& =\frac{d}{d t}\left[\alpha_{t}(Y)\right]-\alpha_{t}\left[\frac{d Y}{d t}\right] .
\end{aligned}
$$

As $Y$ is a fixed vector, this expression reduces to

$$
\frac{d}{d t}\left[\alpha_{t}(Y)\right] .
$$

By (2), this is the same as

$$
\frac{d}{d t}\left[\tau_{t}^{-1}(J(t))\right],
$$


and in turn this is clearly equal to

$$
\tau_{t}^{-1}\left[\frac{d}{d t} J(t)\right]=\tau_{t}^{-1}\left(J^{\prime}(t)\right)
$$

We therefore have

$$
\begin{aligned}
\tau_{t} \circ \alpha_{t}^{\prime} \alpha_{t}^{-1}\left[\tau_{t}^{-1}(J(t))\right] & =\tau_{t} \tau_{t}^{-1}\left(J^{\prime}(t)\right) \\
& =J^{\prime}(t) .
\end{aligned}
$$

Now $J^{\prime}(t)=\nabla_{N(t)} J(t)$ where $N(t)$ is the outward normal to the distance sphere of radius $t$ about $\gamma(0)$. It is clear that $[J, N] \equiv 0$, and it follows that $J^{\prime}(t)=\nabla_{J(t)} N(t)$. By the definition of the shape operator,

$$
\nabla_{J(t)} N(t)=S_{t}(J(t))
$$

We therefore have

$$
\tau_{t} \alpha_{t}^{\prime} \alpha_{t}^{-1} \tau_{t}^{-1}(J(t))=S_{t}(J(t))
$$

for all $t$, and in particular when $t=t_{0}$ we have

$$
\tau_{t_{0}} \alpha_{t_{0}}^{\prime} \alpha_{t_{0}}^{-1} \tau_{t_{0}}^{-1}(X)=S_{t_{0}}(X),
$$

and the result is proved.

As the mean curvature is just the trace of the shape operator, we obtain the following corollary.

COROLlary 2. The mean curvature $m(t)$ of the distance sphere about $\gamma(0)$ at $\gamma(t)$ is given by

$$
m(t)=\operatorname{trace} A_{t}^{\prime} A_{t}^{-1} .
$$

We now come to the main result of this section.

Proposition 1. Let $M^{n}$ be a complete, non-compact Riemannian manifold, and let $\gamma(t), t \geqslant 0$, be a geodesic originating at a point $p \in M$. Suppose that $\operatorname{Ric}(\dot{\gamma}(t)) \geqslant 0$ for all $t$. Suppose further that

$$
\operatorname{Ric}(\dot{\gamma}(t)) \geqslant(n-1) \delta>0
$$

for all $t \in[0, R]$. Then, if $R>\pi / 2 \sqrt{\delta}$, there is a point along $\gamma$ conjugate to $p$. In particular, $\gamma$ is not a ray.

Proof. We establish the proposition by contradiction.

Suppose that we can find a geodesic $\gamma$ which satisfies the hypotheses, but which contains no point conjugate to $p$. This is equivalent to saying that the matrix $A_{t}$ is non-singular for all $t>0$. It follows that $\operatorname{tr} A_{t}^{\prime} A_{t}^{-1}(t)$ is well-defined and smooth (because Jacobi fields are smooth) for all $t>0$. By Corollary 2, this quantity is just the mean curvature of the distance sphere of radius $t$ from $p$ at the point $\gamma(t)$. It follows that $m$ must be finite for all $t>0$.

Now consider the curvature requirement. By Corollary 1, we know that

$$
m \leqslant(n-1) \sqrt{\delta} \tan \left(\frac{\pi}{2}-t \sqrt{\delta}\right)
$$

for all $0<t \leqslant R$. The quantity on the right-hand side is just the mean curvature 
of the distance sphere of radius $t$ in the space form of constant curvature $\delta$. For $t>\pi / 2 \sqrt{\delta}$ this is clearly negative, and so we conclude that $m(t)<0$ also.

By Lemma 3, it follows that there exists $t_{1}$ such that

$$
\lim _{t \rightarrow t_{1}+} m(t)=-\infty .
$$

However, we have just established that $m(t)$ is finite for all $t>0$, and hence we have our contradiction.

From Proposition 1 we easily obtain a further corollary.

COROLlary 3. Let $M^{n}$ be a complete Riemannian manifold with non-negative Ricci curvature. Suppose there exists a geodesic ball $B_{p}(R) \subset M$ with centre $p$ and radius $R$ such that

$$
\left.\operatorname{Ric}\right|_{B_{p}(R)} \geqslant(n-1) \delta>0 .
$$

If $R>\pi / 2 \sqrt{\delta}$, then $M$ is compact.

According to the well-known Bonnet-Myers theorem, if the Ricci curvature satisfies (3) for $R \geqslant \pi / \sqrt{\delta}$, then $M$ is compact. In Corollary 3 , we weaken this assumption under the additional condition that the Ricci curvature is everywhere non-negative.

REMARK. The result of Ambrose [1] states that the integral of the Ricci curvature along any ray must be finite. (No assumption is made about the Ricci curvature either on the manifold as a whole, or along the ray.) By integrating the Riccati inequality (1) and using Corollary 1 together with the fact that $m(t) \geqslant 0$ for all $t$ (which follows from the proof of Proposition 1), we can easily obtain an alternative proof of the Ambrose result in the special case where the Ricci curvature along the ray is non-negative.

\section{The proof of Theorem A}

In this section we establish Theorem A. First, however, we need some technical lemmas.

Lemma 5. Define functions $y_{i}(t), i=1,2, t \geqslant 0$, by

$$
\begin{aligned}
& y_{i}^{\prime}(t)=-(n-1) \delta-\frac{y^{2}(t)}{n-1}, \\
& y_{i}\left(t_{i}\right)=c,
\end{aligned}
$$

where $t_{2}=t_{1}+\varepsilon$, for some $\varepsilon \geqslant 0$. Then $y_{1}(t)=y_{2}(t+\varepsilon)$ for all $t \geqslant 0$.

Proof. The result is evident from the explicit form of $y_{1}$ and $y_{2}$; see Lemma 1.

Lemma 6. Suppose that we define functions $\zeta_{i}(t), i=1,2$, by

$$
\begin{aligned}
\zeta_{i}^{\prime}(t) & =-(n-1) \delta-\frac{\zeta^{2}(t)}{n-1}, \\
\zeta_{i}\left(t_{0}\right) & =c_{i},
\end{aligned}
$$

for some $t_{0}$. If $c_{1} \geqslant c_{2}$, then for all $t \geqslant t_{0}$ we have $\zeta_{1}(t) \geqslant \zeta_{2}(t)$. 
Proof. The result is a trivial consequence of Lemma 1 and the fact that $\tan ^{-1}(x)$ is an increasing function for all $x$.

Proof of Theorem A. Suppose that $\operatorname{Ric}(\dot{\gamma}(t)) \geqslant(n-1) \delta$ on closed intervals

$$
I_{i}=\left[a_{i}, b_{i}\right]
$$

where $b_{i} \leqslant a_{i+1}$ and $i \geqslant 1$.

Note that these intervals - and any countable union of them - are Lebesgue measurable.

On each interval, define functions $z_{i}$ inductively by

$$
\begin{aligned}
z_{i}^{\prime}(t) & =-(n-1) \delta-\frac{z^{2}(t)}{n-1}, \\
z_{1}\left(a_{1}\right) & =m\left(a_{1}\right), \\
z_{i}\left(a_{i}\right) & =z_{i-1}\left(b_{i-1}\right) .
\end{aligned}
$$

Here, as usual, $m$ denotes the mean curvature along $\gamma(t)$. By Lemma 2 we have $z_{1}\left(b_{1}\right) \geqslant m\left(b_{1}\right)$.

Suppose now that $z_{k}\left(b_{k}\right) \geqslant m\left(b_{k}\right)$. Since $m^{\prime}(t) \leqslant 0$ for all $t$, we have

$$
z_{k+1}\left(a_{k+1}\right)=z_{k}\left(b_{k}\right) \geqslant m\left(b_{k}\right) \geqslant m\left(a_{k+1}\right)
$$

and, applying Lemma 2 again, we see that

$$
z_{k+1}\left(b_{k+1}\right) \geqslant m\left(b_{k+1}\right) \text {. }
$$

It now follows by induction that $z_{i}\left(b_{i}\right) \geqslant m\left(b_{i}\right)$ for all $i$.

Let $\Delta_{k}=\sum_{i=1}^{k}\left(b_{i}-a_{i}\right)$. In other words, $\Delta_{k}=\sum_{i=1}^{n}\left|I_{i}\right|$.

We want to compare the $z_{i}(t)$ with the function $z(t)$ from Definition 1 . There are two cases to consider.

Case 1: If $t_{0}=0$ and $c=z_{1}\left(a_{1}\right)$ in the definition of $z$, then $z\left(\Delta_{k}\right)=z_{k}\left(b_{k}\right)$. This is a consequence of Lemma 5 .

Case 2: If $t_{0}=0$ and $c=\infty$, then $z\left(\Delta_{k}\right) \geqslant z_{k}\left(b_{k}\right)$ (by Case 1 and Lemma 6).

Either way, we find that $z\left(\Delta_{k}\right) \geqslant m\left(b_{k}\right)$.

From the explicit formula for $z$ (Lemma 1) using $t_{0}=0$ and $c=\infty$, we see that if $\Delta_{k}>\pi / 2 \sqrt{\delta}$ for some $k$, then $z\left(\Delta_{k}\right)<0$ and therefore $m\left(\Delta_{k}\right)<0$. By the proof of Proposition 1, it follows that $\gamma(t)$ contains a point conjugate to $\gamma(0)$. Since this would contradict our assumption that $\gamma$ is a ray, we conclude that $\Delta_{k} \leqslant \pi / 2 \sqrt{\delta}$ for all $k$. Defining $I_{\delta}=\sup _{k} \Delta_{k}$, we therefore have $I_{\delta} \leqslant \pi / 2 \sqrt{\delta}$.

Finally, we must exclude the possibility that $I_{\delta}=\pi / 2 \sqrt{\delta}$.

Suppose that there is only one interval $\left[a_{1}, b_{1}\right]$ on which $\operatorname{Ric}(\dot{\gamma}(t)) \geqslant(n-1) \delta$. If the length of this interval is $\pi / 2 \sqrt{\delta}$, then it follows from the above that $m(t)=0$ for all $t \geqslant b_{1}$. We conclude from the Riccati inequality (1) that $\operatorname{Ric}(\dot{\gamma}(t))=0$ for all $t \geqslant b_{1}$. However, $\operatorname{Ric}(\dot{\gamma}(t)) \geqslant(n-1) \delta$ for all $t \in\left[a_{1}, b_{1}\right]$, a contradiction.

Suppose now there are at least two such intervals. As

$$
\operatorname{Ric}\left(\dot{\gamma}\left(b_{1}\right)\right)=\operatorname{Ric}\left(\dot{\gamma}\left(a_{2}\right)\right)=(n-1) \delta>0,
$$

this means there is a point $x \in\left(b_{1}, a_{2}\right)$ for which $\operatorname{Ric}(\dot{\gamma}(x))>0$. Thus $z_{2}\left(a_{2}\right)-m\left(a_{2}\right)=\varepsilon$ for some $\varepsilon>0$ as a consequence of (1), and by Lemma 2 and Lemma 6 it is easy to see that $z_{k}\left(b_{k}\right)-m\left(b_{k}\right) \geqslant \varepsilon$ for all $k \geqslant 2$. We deduce that $z\left(\Delta_{k}\right)-m\left(b_{k}\right) \geqslant \varepsilon$ for all $k \geqslant 2$, and so $z\left(\Delta_{k}\right) \geqslant \varepsilon$ as the mean curvature is non-negative. However, if $I_{\delta}=\pi / 2 \sqrt{\delta}$, we must have $\lim _{k \rightarrow \infty} z\left(\Delta_{k}\right)=0$, another contradiction. Therefore $I_{\delta}<\pi / 2 \sqrt{\delta}$, and Theorem $\mathrm{A}$ is proved. 


\section{References}

1. W. Ambrose, 'A theorem of Myers', Duke Math. J. 24 (1957) 345-348.

2. J. CHEEGER, Critical points of distance functions and applications to geometry, Lecture Notes in Math. 1504 (Springer, New York, 1991) 1-38.

3. W. KlingenberG, Riemannian geometry, de Gruyter Stud. in Math. 1 (De Gruyter, Berlin, 1982).

David Wraith

Department of Mathematics

National University of Ireland

Maynooth

Co. Kildare

Ireland

dwraith@maths.may.ie 
Copyright of Bulletin of the London Mathematical Society is the property of Oxford University Press / USA and its content may not be copied or emailed to multiple sites or posted to a listserv without the copyright holder's express written permission. However, users may print, download, or email articles for individual use. 